\title{
FATORES INTERVENIENTES NA IMPLANTAÇÃO DO CHECKLIST DE CIRURGIA SEGURA EM UM HOSPITAL UNIVERSITÁRIO
}

Rosilene Alves Ferreiraㄹ, Ricardo de Oliveira Meneses², Cintia Silva Fassarella², Maria Virgínia Godoy da Silva', Gláucya Lima Daú ${ }^{3}$

Objetivo: identificar os fatores intervenientes na implantação do checklist de cirurgia segura em um hospital universitário. Metodologia: Estudo descritivo, exploratório, com abordagem qualitativa e análise de conteúdo de Bardin. Coleta de dados realizada no período de março a maio de 2017, por meio de entrevistas posteriormente transcritas na íntegra. População: três enfermeiros, quatro residentes de enfermagem, sete residentes de medicina e nove técnicos de enfermagem. Resultados: foram agrupados em dois eixos - os fatores que dificultam a implantação do checklist, sendo estes, modificar a cultura, déficit de material, resistência da equipe e a burocracia; e os fatores que favorecem, como o fato de ser hospital universitário e a qualificação profissional. Conclusão: A mudança cultural destacou-se como dificultador no processo de implantação do checklist de cirurgia segura, em contrapartida, o principal fator que favorece esse processo é a instituição ser unidade de ensino tendo o corpo acadêmico inserido na assistência.

Palavras-chave: Lista de checagem, Centro cirúrgico, Segurança do paciente, Enfermagem.

\section{INTERVENING FACTORS IN THE IMPLANTATION OF THE SAFE SURGERY CHECK LIST IN A UNIVERSITY HOSPITAL}

Objective: to identify the factors involved in implanting the safe surgery checklist in a university hospital. Methodology: Descriptive, exploratory study with a qualitative approach. Data collection was carried out from March to May 2017, through interviews later transcribed in full. Three nurses, four nursing residents, seven medical residents and nine nursing technicians participated in the study. For the data treatment, Bardin content analysis was used. Results: were grouped into two axes - the factors that make it difficult to implement the checklist, being these, modify the culture, material deficit, team resistance and bureaucracy; and the factors that favor it, such as being a university hospital and professional qualification. Conclusion: The cultural change was highlighted as a difficult factor in the implementation process of the safe surgery checklist, in contrast, the main factor that favors this process is the institution being a teaching unit having the academic body inserted in the care. Key words: Checklist, Surgicenter, Patient safety, Nursing.

\section{FACTORES INTERVINIENTES EN LAIMPLANTACIÓN DELCHECKLISTDE CIRUGÍASEGURAEN UN HOSPITALUNIVERSITARIO}

Objetivo: identificar los factores intervinientes en la implantación del checklist de cirugia segura en un hospital universitario. Metodología: Estudio descriptivo, exploratorio, con abordaje cualitativo. La recolección de datos realizada en el período de marzo a mayo de 2017, por medio de entrevistas posteriormente transcritas en su totalidad. Participaron del estudio tres enfermeros, cuatro residentes de enfermería, siete residentes de medicina y nueve técnicos de enfermería. Para el tratamiento de los datos se utilizó el análisis de contenido de Bardin. Resultados: fueron agrupados en dos ejes - los factores que dificultan la implantación del checklist, siendo éstos, modificar la cultura, déficit de material, resistencia del equipo y la burocracia; y los factores que favorecen, como el hecho de ser hospital universitario y la calificación profesional. Conclusión: El cambio cultural se destacó como dificultador en el proceso de implantación del checklist de cirugía segura, en contrapartida, el principal factor que favorece ese proceso es la institución ser unidad de enseñanza teniendo el cuerpo académico insertado en la asistencia. Palabras clave: Lista de Verificación, Centro quirúrgico, Seguridad del paciente, Enfermería.

1Universidade do Estado do Rio de Janeiro, UERJ.

'Universidade do Grande Rio, UNIGRANRIO. UERJ.

${ }^{3}$ Universidade Federal do Estado do Rio de Janeiro, UNIRIO.

Autor correspondente: Rosilene Alves Ferreira. E-mail: rosilene_alves@ymail.com 


\section{INTRODUÇÃO}

A Organização Mundial da Saúde (OMS) em parceria com a Universidade de Harvard criou campanhas para se efetivar as práticas instituídas no segundo desafio global "Cirurgias Seguras Salvam Vidas". Para isso, adotou-se um checklist composto por três partes: Identificação (antes da indução anestésica), Confirmação (antes da incisão cirúrgica) e Registro (antes de o paciente sair da sala cirúrgica)(1).

A Identificação consiste em confirmar os dados do paciente, se houve ou não se aplica demarcação do sítio cirúrgico, a segurança anestésica, a oximetria de pulso em funcionamento, se o paciente possui alergias, via aérea difícil e equipamento de assistência disponivel e, se há risco de perda sanguínea(1-2).

Já a Confirmação tem por objetivo garantir que todos os membros da equipe se conheçam, confirmar novamente o paciente, o sítio cirúrgico e o procedimento proposto, revisar momentos críticos previstos pela equipe cirúrgica, anestésica e de enfermagem, confirmar a administração, ou se não se aplica, do antimicrobiano profilático ${ }^{(1-2)}$.

No Registro o profissional responsável confirma verbalmente se foi feito o registro completo do procedimento realizado, se as contagens de instrumentais cirúrgicos, compressas e agulhas estão corretas, identificação correta das amostras para anatomia patológica, se ocorreu algum problema com equipamentos durante a cirurgia e, toda a equipe revisa preocupações essenciais para a recuperação do paciente ${ }^{(1-2)}$.

O checklist tem trazido resultados e retorno para a prática clínica devendo ser utilizado de forma bem-sucedida em diversos contextos cirúrgicos, mesmo reconhecendo que sua implantação e adesão por parte dos profissionais requer comprometimento, adaptação e mudança de paradigma $a^{(2-3)}$.

A motivação para este estudo surgiu enquanto residente em enfermagem do Centro Cirúrgico, Centro de Material e Esterilização e Recuperação Anestésica - CC/CME/RA de um Hospital Universitário a partir de observações de campo de problemas nos atos cirúrgicos com a identificação do paciente, com a presença de alergias, com a necessidade de uso de hemoderivados, entre outros.

Sendo assim, constitui-se como questão de pesquisa: quais os fatores que são percebidos pela equipe cirúrgica que influenciam na implantação do checklist de cirurgia segura no hospital universitário? Tendo por objeto de estudo os fatores que dificultam e favorecem o processo de implantação do checklist de cirurgia segura, tendo por objetivo identificar e discutir tais fatores.

Um estudo piloto, realizado em 2008 e publicado no New England Journal of Medicine, comparou antes e depois da utilização do checklist em 7.688 pacientes e comprovou a redução em $36 \%$ de grandes complicações e em $47 \%$ de mortalidade, com significância estatística ${ }^{(4-5)}$.

No ano seguinte, a OMS estimou uma diminuição de 500 mil óbitos após a aplicação do checklist de cirurgia segura. A aplicação de uma simples lista demonstrou redução significativa da incidência de grandes complicações ${ }^{(5)}$.

Contudo, ainda são necessários estudos qualitativos e quantitativos sobre o processo de implantação do checklist de cirurgia segura, considerando a realidade de cada instituição, desde a obrigatoriedade da RDC 36/13 que dispõe sobre a segurança do paciente.

Diante do exposto, o objetivo deste estudo foi identificar os fatores intervenientes na implantação do checklist de cirurgia segura em um hospital universitário.

Este trabalho justifica-se por ser comprovada a eficácia do checklist de cirurgia segura tornando-se importante aos profissionais e estudantes de saúde, que se dedicam a sua formação e aperfeiçoamento no que refere à realização das etapas para uma cirurgia segura e seu processo de implantação e estruturação.

\section{METODOLOGIA}

\section{Tipo de estudo}

Trata-se de um estudo descritivo e exploratório, com abordagem qualitativa.

\section{Participantes da pesquisa}

A população do estudo é composta por profissionais médicos cirurgiões, médicos residentes de cirurgia e anestesia, enfermeiros, residentes de enfermagem e técnicos de enfermagem. A população delimitou-se pela abrangência dos atores envolvidos, não visando à quantificação das informações e sim a qualidade do conteúdo devido à extensão do objeto e a complexidade do estudo. Adotouse como critérios de inclusão: profissionais da assistência transoperatória; experiência mínima de um ano; residentes de enfermagem e de medicina. Foram excluídos residentes do 1 으 ano devido ao menor contato com o cenário e funcionários administrativos.

\section{Local do estudo}

O cenário foi o Centro Cirúrgico de um Hospital Universitário no estado do Rio de Janeiro com nove salas operatórias ativas atendendo a cirurgias de pequeno, médio e grande porte, realizando cerca de 650 cirurgias/mês no período de março a abril.

\section{Coleta dos dados}

Após a abordagem dos sujeitos, a coleta de dados foi 
realizada no Centro Cirúrgico, sem que interferisse na dinâmica de atendimento da unidade, por meio de entrevistas que foram gravadas e salvas em mp3, sendo transcritas na integra posteriormente pela autora, com revisão do texto escrito nos depoimentos pelos participantes.

Para a entrevista utilizou-se um roteiro composto de duas etapas. A primeira, destinada à identificação dos sujeitos contendo as seguintes variáveis: cargo, idade, sexo, tempo de experiência profissional, tempo de experiência em centro cirúrgico e tempo de experiência em hospital universitário.

A segunda etapa compôs-se de questões específicas referentes aos fatores intervenientes na implantação do checklist de cirurgia segura:(1) $\bigcirc$ que você sabe sobre o checklist de cirurgia segura e as etapas que o compõe? ${ }^{(2)} \mathrm{Na}$ sua concepção, quais os fatores existentes, em um hospital universitário, que dificultam a implantação do checklist de cirurgia segura?(3) $\mathrm{Na}$ sua concepção, quais os fatores existentes, em um hospital universitário, que favorecem a implantação do checklist de cirurgia segura? ${ }^{(4)}$ Se você fosse passar por uma cirurgia, gostaria que o checklist fosse aplicado?

\section{Procedimento de análise dos dados}

Para o processo de análise e discussão dos dados foi adotado o método de análise de conteúdo de Bardin. A finalidade dessa análise é a inferência de conhecimentos relativos às condições dos dados produzidos ${ }^{(6,7)}$.

Esta abordagem compõe-se em três etapas: a pré-análise, a exploração do material e o tratamento dos resultados. A decodificação e interpretação dos dados foram definidas por categorias semânticas classificadas após a transcrição das entrevistas, utilizando fragmentos de falas semelhantes para análise temática, em que os dados foram tratados de modo a serem significativos e válidos ${ }^{(7)}$

A pré-análise constituiu-se em organizar e estruturar as ideias preparando uma sintese das falas na íntegra dos sujeitos e, lendo os textos, exaustivamente, buscando uma representatividade nas entrevistas realizadas.

$\mathrm{Na}$ exploração do material os dados brutos foram transformados em características relevantes para a classificação das categorias.

A decodificação e interpretação dos dados foram definidas por categorias semânticas classificadas após a transcrição das entrevistas utilizando fragmentos de falas semelhantes para análise do conteúdo.

As falas dos sujeitos foram codificadas pelas seguintes letras: "A" para os Anestesiologistas, "C" para o Cirurgião e "E" para os membros da Enfermagem.

No Tratamento dos resultados, os dados brutos foram tratados de maneira a propor inferências e adiantar interpretações sobre os objetivos previstos ou descobertas inesperadas, confrontando os resultados obtidos com o material servindo de base para a análise.

A partir dessa análise, foram entrevistados 24 sujeitos, que além de proporcionar a qualidade do estudo, forneceu a quantidade consensual para uma pesquisa qualitativa que, segundo Morse e Creswell são de vinte a trinta entrevistas ${ }^{(6)}$.

\section{Procedimentos éticos}

Quanto aos aspectos éticos e legais, primeiramente, o projeto de pesquisa foi encaminhado a Chefia médica e de enfermagem do Centro Cirúrgico para análise e concordância. Após a autorização, o projeto foi encaminhado à Direção Geral e Coordenadoria de Enfermagem, tendo a anuência conforme Declaração instituída pelo Comitê de ética da Instituição. O projeto foi submetido à apreciação do Comitê de Ética em Pesquisa da unidade com o Parecer Consubstanciado número 64147716.7.0000.5259.

Após a assinatura do Terno de Consentimento Livre e Esclarecido (TCLE), foram utilizadas para análise dos dados as falas de 09 técnicos de enfermagem, 03 enfermeiros, 04 residentes de enfermagem e 07 residentes de medicina, totalizando 23 discursos.

Cabe ressaltar que houve recusas para participação no estudo, dentre as quais se encontram médicos cirurgiões e um residente de cirurgia plástica, que solicitou a retirada de sua entrevista da análise dos dados. Sendo assim, foram analisadas 23 entrevistas.

\section{RESULTADOS}

Dos 23 sujeitos entrevistados, houve uma população predominantemente feminina (64,00\%) e idade média de 40 anos. Em relação à experiência profissional, a tabela 1 demonstra tal distribuição.

Tabelal - Distribuição da experiência profissional dos participantes. Rio de Janeiro, 2017.

\begin{tabular}{c|c|c|c}
\hline \multicolumn{4}{c}{ Experiência profissional } \\
\hline Tempo & $\begin{array}{c}\text { Experiência profissional } \\
(\%)\end{array}$ & $\begin{array}{c}\text { Experiência em Centro } \\
\text { Cirúrgico (\%) }\end{array}$ & $\begin{array}{c}\text { Experiência na } \\
\text { instituição (\%) }\end{array}$ \\
\hline 1 a 5 anos & 47,82 & 47,82 & 69,56 \\
\hline $6-10$ anos & 4,34 & 17,39 & 4,34 \\
\hline $11-15$ anos & 4,34 & 6,69 & - \\
\hline 16 anos ou mais & 43,50 & 26,10 & 26,10 \\
\hline
\end{tabular}

Quanto aos fatores intervenientes na implantação do checklist de cirurgia segura, com a análise temática foi possivel identificar dois eixos temáticos: fatores que dificultam e fatores que favorecem na implantação do checklist de cirurgia segura em um hospital universitário. 
Eixo A - Fatores que dificultam na implantação do checklist de cirurgia segura. Este grupo foi dividido em quatro subcategorias.

Subcategoria 1 - Ausência de uma cultura de segurança

Em 40,00\% das falas dos sujeitos o principal dificultador para a implantação do checklist de cirurgia segura tratouse de uma questão cultural. Tal fato é expresso nas falas abaixo."A questão da cultura mesmo. Eles trabalham muito tempo desse jeito e não querem tentar uma nova coisa." (E2)

"Acho que é uma questão institucional, sabe". (A20)

\section{Subcategoria 2- Déficit de material}

Foi identificado em $23,00 \%$ das falas dos sujeitos que a falta de produtos para saúde e de instrumentais cirúrgicos dificultam a execução do checklist.

"A falta de material né." (E5)

"Questão de falta de material." (El6)

\section{Subcategoria 3 - Resistência da equipe}

Essa subcategoria é composta por $20,00 \%$ dos sujeitos entrevistados. A resistência por parte da equipe se torna um inviabilizador para que o checklist seja implantado eficazmente.

"Os médicos. Principalmente os médicos, eles são muito resistentes a isso." (Ell)

"Teria que encher o saco dos profissionais. Tem que, sei lá, de alguma forma tem que obrigá-los a fazer." (A19)

\section{Subcategoria 4 - Burocracia}

Em 17,00\% das falas as palavras "papel" e "sobrecarga" foram citadas dando origem a esta subcategoria.

"O que complica mesmo é a quantidade de papéis [...] aí esse mais um é um problema." (A7)

"Mas acho que dificulta principalmente porque ele teria que ser empregado por funcionários que já estão sobrecarregados ali naquele início da cirurgia." (C23)

Eixo B - Fatores que favorecem na implantação do checklist de cirurgia segura

Este grupo foi dividido em duas subcategorias: "hospital universitário" e "qualificação profissional".

\section{Subcategoria 1 - Hospital universitário}

Representado por $70,00 \%$ das falas com a palavra "hospital universitário".

"O próprio fato de ser um hospital universitário." (E4)

"Além de ser um hospital acadêmico [...], que dar tempo de fazer tudo bem organizado". (C23)
Subcategoria 2 - Qualificação profissional

Representada por $30 \%$ das falas dos sujeitos, segue os fragmentos:

"Aqueles que são interessados por uma assistência de qualidade". (E3)

"O que favorece a implantação do checklist é pessoal capacitado, vários professores, bastantes graduados que podem complementar e ensinar o checklist, tem o corpo de alunos e tudo mais". (C23)

\section{DISCUSSÃO}

A média de idade foi de 40 anos, mesmo sendo $47,82 \%$ residentes.

O sexo predominante foi o feminino, o que não contraria o contexto da enfermagem, uma vez que o trabalho é exercido quase exclusivamente por mulheres, sendo explicado pela própria retrospectiva da profissão(8)

O tempo de experiência profissional, em centro cirúrgico e na instituição foi predominante de 1 a 5 anos, pois dos 23 sujeitos, 11 (47,82\%) eram residentes tendo o seu primeiro contato no mercado de trabalho em que estudam para se especializarem em determinada área.

A partir das falas dos sujeitos foram identificados como intervenientes na implantação do checklist de cirurgia segura foram agrupados em dois eixos.

No primeiro eixo, os fatores intervenientes são os que dificultam no processo de implantação do instrumento sendo estes a ausência de cultura de segurança, o déficit de material, a resistência da equipe e a burocracia.

Quanto à cultura de segurança, diversos estudos demonstraram que esta dificulta na execução de estratégias visando à segurança do paciente..$^{(5,9-11)}$.

Uma pesquisa realizada em um hospital universitário no Brasil destacou que alguns profissionais não estavam preocupados com a mudança em seu comportamento para a realização do checklist, corroborando com os mesmos achados deste estudo. ${ }^{(12)}$.

Considerando o cenário complexo do que é o centro cirúrgico, a cultura de segurança ganha destaque, pois se percebe que os valores individuais e de grupo, influenciam a prática profissional ${ }^{(13,14)}$. Este destaque pode ser identificado conforme incidência no levantamento deste estudo.

Quanto ao déficit de material, a ausência dos materiais adequados para a realização das cirurgias causa dificuldade na implantação do checklist. Entretanto, o uso da lista auxilia na identificação da falta desses produtos permitindo demonstrar ao gestor o que é necessário adquirir para garantir a segurança do paciente cirúrgico(14,15).

É importante destacar que a identificação deste fator foi levantada pela enfermagem uma vez que essa categoria 
é responsável pelo preparo, solicitação e conferência dos recursos materiais inerentes à cirurgia

Em relação à resistência profissional, esta também foi observada em outro estudo com hospitais ingleses que evidenciou dificuldade na adesão ao checklist entre médicos seniores ${ }^{(12)}$. Este achado corroborou com o presente estudo, pois houve recusa dos médicos staffs abordados demonstrando resistência à temática segurança cirúrgica.

Nesse contexto, vale ressaltar que é importante que haja quebra de paradigmas de hierarquia e falta de comprometimento, reconhecendo que a segurança é um critério básico para garantir a qualidade da assistência ao paciente ${ }^{(16-17)}$

Por último, a burocracia foi expressa em um entendimento de que o checklist é "mais um papel a ser preenchido".

O resultado de uma avaliação em oito instituições pilotos mostrou que o uso do checklist praticamente dobrou a chance dos usuários receberem o tratamento cirúrgico com padrões de cuidado adequados. Nestes locais, houve uma redução de $47 \%$ da mortalidade e as complicações, que eram em média $11 \%$, passou a ser de $7 \%(1,18-19)$.

Portanto, o checklist de cirurgia segura não deve ser considerado um procedimento meramente burocrático, conforme descrito na quarta subcategoria. Para isso, a equipe necessita maximizar as condições favoráveis à implantação do protocolo.

O segundo eixo identificou os fatores que favorecem a implantação do checklist de cirurgia segura, sendo estes, a característica singular da instituição em ser um hospital universitário e a qualificação profissional.

Em relação à característica de ensino do hospital, foi considerado favorável por ser entendido como unidade de cuidado especializado na assistência, além de ser unidade formadora de novos profissionais através dos programas de residência médica/multiprofissional, tendo doutores inseridos na assistência.

Este achado corrobora com o presente estudo, pois os sujeitos entendem que o fato de ser um hospital universitário, facilita a execução de novos instrumentos desenvolvidos por universidades e comprovados sua eficácia em estudos científicos além de formar profissionais com a segurança do paciente internalizado em sua prática.

A segurança do paciente necessita ser problematizada e discutida sendo indispensável instrumentalizar os futuros profissionais para a prevenção de eventos adversos e desenvolver neles a cultura da segurança do paciente ${ }^{(20)}$.

Nesse mesmo entendimento, a qualificação profissional se encontra presente, pois o egresso ao hospital universitário é apoiado através de processo seletivo, o que seleciona os profissionais que estarão inseridos no corpo profissional.
Em se tratando de um hospital universitário, a seleção de pessoas aptas ao acompanhamento dos acadêmicos e compartilhamento de conhecimentos teórico-práticos atualizados é fundamental, impondo que as instituições implantem estratégias que visem à seleção de profissionais que melhor cumpram as tarefas dos cargos a serem ocupados $(8,20)$

Portanto, a implantação do checklist em um hospital universitário torna-se favorável, pois, além do corpo acadêmico encontrar-se inserido na assistência, há profissionais atualizados, qualificados e dispostos a implantar o checklist.

\section{Limitações do estudo}

O período de coleta dos dados coincidiu com um período de crise econômica onde o hospital manteve suas atividades em contingenciamento de cirurgias e funcionários, o que influenciou no tamanho amostral da pesquisa. Entretanto não interferiu na representatividade para o tratamento dos dados.

\section{Contribuições do estudo para a prática}

Este estudo proporciona mudanças de paradigmas internalizadas pelos profissionais de saúde atuantes nesta unidade. Uma vez identificado o que dificulta e o que favorece a implantação do Programa Cirurgias Seguras Salvam Vidas, basta iniciativa para sua efetiva implementação a partir de treinamentos e execução de tarefas.

\section{CONSIDERAÇÕES FINAIS}

Os fatores identificados pelos sujeitos deste estudo como dificultadores devem ser utilizados como ponto de partida para a mudança do modo de agir dos profissionais e da gestão hospitalar em questão, a fim de se implantar tal instrumento devido a sua importância.

A partir dos resultados encontrados no presente estudo, a mudança cultural foi destacada como o principal dificultador para a implantação do checklist de cirurgia segura em um hospital universitário.

Foi considerado pelos participantes deste estudo em sua unanimidade que o fato de ser um hospital universitário favorece a implantação do programa por ser unidade base de formação de profissionais qualificados, o que possibilita uma mudança cultural.

É imprescindivel, para o paciente submetido a uma assisıtência cirúrgica, que os procedimentos anestésicocirúrgicos ocorram com melhor qualidade e os possiveis erros sejam mitigados por uma cultura de segurança do paciente cirúrgico.

Considerando o hospital unidade formadora de profissionais, como é possivel continuar moldando futuros 
trabalhadores sem a prática comprovada em diversos estudos nacionais e internacionais da eficácia do checklist de cirurgia segura?

Portanto, é possivel inferir que o maior desafio para a implantação do checklist de cirurgia segura no hospital universitário estudado é o de transformar a segurança do paciente cirúrgico em prioridade estratégica, por entender que segurança é investimento, focando em medidas de prevenção.

\section{Contribuições dos autores}

Concepção e/ou desenho: Ferreira RA; Analise e interpretação dos dados: Ferreira RA, Meneses RO e Fassarella CS; Redação do artigo: Ferreira RA, Meneses RO e Fassarella CS:

Revisão critica: Meneses RO, Fassarella CS, Silva MVG e Daú GL; Revisao final: Meneses RO e Fassarella CS.

\section{REFERÊNCIAS}

1. Ministério da Saúde (BR), Agência Nacional de Vigilância Sanitária, Organização Mundial da Saúde. Segundo desafio global para a segurança do paciente. Cirurgias seguras salvam vidas. Rio de Janeiro: Organização Pan-Americana de Saúde: 2009. [Acesso em 2017 Mar 23]. Disponivel em: http://bvsms.saude.gov.br/bvs/publicacoes/seguranca_paciente_cirurgias_seguras_salvam_vidas.pdf

2. Corona ARPD, Peniche ACG. A cultura de segurança do paciente na adesão ao protocolo da cirurgia segura. REV. SOBECC. Săo Paulo. jul./set. 2015[Acesso em protocolo da cirurgia segura. REV. SOBECC. Sao Paulo. jul./set. 2015[Acesso em
2017 Nov 21]: 20(3): 179-185. Disponivel em: http://www.sobecc.org.br/arquivos/ artigos/2015/pdfs/v20n3/179-185.pdf

3. Sociedade Brasileira de Enfermeiros de Centro Cirürgico, Recuperação Anestésica e Centro de Material e Esterilização (SOBECC). Práticas recomendadas: centro cirúrgico, recuperação pós-anestésica, centro de material e esterilização. $7{ }^{\sharp}$ ed. São Paulo: SOBECC: 2017

4. Freitas MR, Antunes AG, Lopes BNA, Fernandes FC, Monte LC, Gama ZAS. Avaliação da adesão ao checklist de cirurgia segura da OMS em cirurgias urológicas e ginecológicas, em dois hospitais de ensino de Natal, Rio Grande do Norte. Brasil. Cad. Saúde Pública [Periódico na Internet] 2014 Jan [Acesso em 2017 Nov 03]; 30(1): 137-48. Disponivel em: http://www.scielosp.org/scielo.php?script=sci_arttextEpid=S0102311X2014000100137\&lng=en. http://dx.doi.org/10.1590/ $0102-311 \times 00184612)$

5. Souza RM; Araújo MGS; Verissimo RCSS; Comassetto I; Ferreira FAZ; Bernardo THL. Aplicabilidade do checklist de cirurgia segura em centros cirúrgicos hospitalares. Rev. SOBECC, São Paulo. out./dez. 2016[Acesso em 2017 Nov 21]; 21(4): 192197. Disponivel em: https://revista.sobecc.org.br/sobecc/article/view/67

6. Minayo. MCS. Revista Pesquisa Qualitativa. São Paulo (SP). v. 5, n. 7. p. 01-12 abril. 2017. [Acesso em 2017 Nov 23]. Disponivel em: https://edisciplinas.usp.br/pluginfile.php/4111455/mod_resource/content/1/Minayosaturacao.pdf.

7. Bardin L. Análise de conteúdo. Lisboa: ediçōes 70. 2011. 229p

8. Machado, Maria Helena(Coord.).Perfil da enfermagem no Brasil: relatório final: Brasil / coordenado por Maria Helena Machado. Rio de Janeiro: NERHUS -DAPS -ENSP/Fiocruz, 2017. [Acesso em 2019 Jun 20] 748p. vol 01. Disponivel em: http:// www.cofen.gov.br/perfilenfermagem/pdfs/relatoriofinal.pdf

9. Pancieri AP. Santos BP. Avila MAG, Braga EM. Checklist de cirurgia segura: análise da segurança e comunicação das equipes de um hospital escola. Rev Gaúcha Enferm. 2013[Acesso em 2017 Nov 22]:34(1):71-78. Disponivel em: http://www.scielo.br/pdf/rgenf/v34nl/09.pdf

10. Maziero ECS, Silva AEBC, Mantovani, MF, Cruz EDA. Adesão ao uso de um checklist cirürgico para segurança do paciente. Rev Gaúcha Enferm.2015[Acesso em 2017 Nov 20]. 36(4):14-20. Disponivel em: https://seer.ufrgs.br/RevistaGauchadeEnfermagem/article/view/53716
11. Porto KLH. A segurança do paciente na utilização do checklist. Enferm rev. 2014 [ Acesso em 2017 Nov 22]; 17(2) 103-115. Disponivel em: http://periodicos.pucminas. br/index.php/enfermagemrevista/article/view/12876

12. Carvalho PA, Göttems LBD, Pires MRGM, Oliveira MLC Cultura de segurança no centro cirúrgico de um hospital público, na percepção dos profissionais de saúde. Rev. Latino-Am. Enfermagem nov.-dez. 2015; [Acesso em 2017 Nov 23] 23(6):1041-8. Disponivel em: http://www.scielo.br/pdf/rlae/v23n6/pt_0104-1169-rlae-23-06-0104l.pdf

13. Zambon LS. Daud-Gallotti R, Novaes HMD: Aliança Mundial para a Segurança da Organização Mundial da Saúde. Organização Pan-Americana da Saúde (OPAS): Ministério da Saúde (BR). Agência Nacional de Vigilância Sanitária (ANVISA); Fundação Oswaldo Cruz, Proqualis. Segundo desafio global: cirurgias seguram salvam vidas.20?? [acesso em 2017 Aug 19]; [aproximadamente 39 p]. Disponivel em: https://proqualis.net/aula/segundo-desafio-global-cirurgias-seguras-salvam-vidas

14. Pancieri AP, Carvalho R, Braga EM. Aplicação do checklist para cirurgia segura: Relato de experiência. Rev SOBECC. 2014[Acesso em 2017 Nov 28]:19(1):26-33. Disponivel em: http://www.sobecc.org.br/arquivos/artigos/2015/pdfs/site_sobecc_v19nl/05_sobecc_v19nl.pdf

15. Gawande A. Checklist: como fazer as coisas bem feitas. Rio de Janeiro: Sextante; 2011

16. Gomes CDPP, Santos AA, Machado ME, Treviso P. Percepção de uma equipe de enfermagem sobre a utilização do checklist cirúrgico. Rev. SOBECC São Paulo. JUL./SET. 2016 [Acesso em 2017 Nov 28]: 21(3): 140-145. Disponivel em: https:// revista.sobecc.org.br/sobecc/article/view/180/pdf

17. Wegner W, Silva SC, Kantorski KJC, Predebon CM, Sanches MO, Pedro ENR. Educação para cultura da segurança do paciente: Implicaçōes para a formação profissional. Escola Anna Nery 20(3) Jul-Set 2016. [acesso em 17 Nov 28]: [aproximadamente $8 \mathrm{p}$ ]. Disponivel em: http://www.lume.ufrgs.br/bitstream/handle/10183/143715/000994839.pdf?sequence $=1$

18. Ministério da Saúde (BR). Hospitais de ensino: o processo de certificação dos hospitais de ensino. [acesso em 2017 Aug 09]: [aproximadamente 03 p] Disponivel em: http://portalsaude.saude.gov.br/index.php/o-ministerio/principal secretarias/964-sas-raiz/dahu-raiz/atencao-hospitalar/l3-atencao-hospitalar/ 14378-certificacao-de-hospitais-de-ensino

19. Alpendre FT, Cruz EDA. Dyniewicz AM, Mantovani MF, Silva AEBC, Santos GS. Safe surgery: validation of pre and postoperative checklists. Rev. Latino-Am. Enfermagem. 2017;25:e2907. [Accesso em 2019 Jun 20]: Disponivel em: http://www. scielo.br/pdf/rlae/v25/pt_0104-1169-rlae-25-e2907.pdf

20. Roscani AN, Ferraz EM, Oliveira Filho AG. Freitas MI. Validação de checklist cirúrgico para prevenção de infecção de sítio cirúrgico Acta Paul Enferm. 2015; [Acesso em 17 Nov 28] 28(6):553-65. Disponivel em: http://www.scielo.br/pdf/ape/ v28n6/1982-0194-ape-28-06-0553.pdf 